\title{
Acupoint Stimulation Using Bee Venom Attenuates Formalin-Induced Pain Behavior and Spinal Cord Fos Expression in Rats
}

\author{
Hyun-Woo KIM ${ }^{1)}$, Young-Bae KWON ${ }^{1)}$, Tae-Won HAM ${ }^{1)}$, Dae-Hyun ROH ${ }^{1)}$, Seo-Yeon YOON ${ }^{1)}$, Hye-Jung LEE ${ }^{2}$, \\ Ho-Jae HAN $^{3)}$, Il-Suk YANG ${ }^{1)}$, Alvin J. BEITZ ${ }^{4)}$ and Jang-Hern LEE ${ }^{1) *}$ \\ ${ }^{1)}$ Department of Veterinary Physiology, College of Veterinary Medicine and School of Agricultural Biotechnology, Seoul National \\ University, Seoul, ${ }^{2}$ Graduate School of East-West Medical Science, Kyung Hee University, Yong-in, ${ }^{3)}$ Hormone Research Center, College \\ of Veterinary Medicine, Chonnam National University, Kwang-ju, South Korea and ${ }^{4}$ Department of Veterinary Pathobiology, College of \\ Veterinary Medicine, University of Minnesota, St Paul, MN, 55108, U.S.A.
}

(Received 6 September 2002/Accepted 20 December 2002)

ABSTRACT. In two previous reports, we have demonstrated that injection of bee venom (BV) into an acupoint produces a significant antinociceptive and anti-inflammatory effect in both a mouse model of visceral nociception and a rat model of chronic arthritis. Th e present study was designed to evaluate the potential antinociceptive effect of BV pretreatment on formalin-induced pain behavior and it associated spinal cord Fos expression in rats. Adult Sprague-Dawley rats were injected with BV directly into the Zusanli (ST36) acupoint or into an arbitrary non-acupoint located on the back. BV pretreatment into the Zusanli acupoint significantly decreased paw-licking time in the late phase of the formalin test. In contrast, BV injected into a non-acupoint in the back region did not suppress the paw-licking time. In addition, BV pretreatment into the Zusanli acupoint markedly inhibited spinal cord Fos expression induced by formalin injection. These findings indicate that BV pretreatment into the Zusanli acupoint has an antinociceptive effect on formalin-induced pain behavior.

KEY WORDS: acupuncture, antinociception, bee venom, formalin test, Fos.

Bee venom (BV) from the honeybee consists of melittin, phospholipase $\mathrm{A}_{2}$, apamin, adolapin, mast cell degranulating peptide and several other bioactive substances [26]. Melittin and phospholipase $\mathrm{A}_{2}$, two major components of $\mathrm{BV}$, are generally thought to play an important role in the induction of the irritation and allergic reaction associated with the bee stings. In this regard, intradermal injection of melittin produces a local hyperthermic effect in human [23]. Phospholipase $\mathrm{A}_{2}$ is a membrane-associated phospholipid converting enzyme that is important in the production of arachidonic acid. Arachidonic acid is further metabolized by one of two enzyme pathways into various prostaglandins (by cyclooxygenase) or leukotrienes (by lipooxygenase). Subcutaneous BV injection into plantar surface of the rat paw produces characteristic pain behaviors including paw licking and flinching [26] while BV administration in cats has been shown to produce prolonged and tonic nociceptive responses associated with changes in the firing of spinal cord neurons [7]. Chen and his colleagues have since published several reports that have elucidated some of the mechanisms underlying BV-induced nociception in rats and cats [7-13, 27, 28, 34, 35].

In contrast to this recent focus on BV's nociceptive effects, Kwon and his co-workers have reported that longterm treatment with BV at a dose of $1 \mathrm{mg} / \mathrm{kg} /$ day produces a significant antinociceptive and anti-inflammatory effect on the Freund's complete adjuvant-induced arthritis in rats [24]. Although natural BV produces irritation when

* Correspondence to: Lee, J.-H., Department of Veterinary Physiology, College of Veterinary Medicine, Seoul National University, Seoul 151-742, South Korea. injected subcutaneously, injection of diluted BV, particularly into an acupoint, can reduce chronic nociception and inflammation. Thus Kwon et al. have shown that BV treatment into an acupoint can significantly reduce arthritisassociated edema and nociceptive responses [24]. In addition, BV injection into the Zhongwan acupoint significantly reduces the number of abdominal stretches induced by intraperitoneal acetic acid injection and also reduces neuronal Fos expression in the spinal cord dorsal horn and nucleus tractus solitarii in mice [25]. In this latter study, BV treatment into a non-acupoint (i.e. on the back) failed to produce this antinociceptive effect except at the highest dose $(2.5$ $\mathrm{mg} / \mathrm{kg}$ ) tested.

In oriental medicine, acupuncture is one of the most common therapies used to treat a number of human inflammatory diseases including rheumatoid arthritis [3] and osteoarthritis [16]. Depending upon the type of acupoint stimulation, acupuncture can be classified into acupressure, manual acupuncture, electroacupuncture and moxibustion. Generally, it is believed that the analgesic effect of acupuncture is due to activation of the descending pain modulating system [21]. Acupoint stimulation is thought to activate primary afferent fibers which in turn stimulate ascending pathways that ultimately activate the descending pain modulating system including the periaqueductal grey (PAG), raphe magnus (RMg) and the locus coeruleus (LC). Endogenous opioids such as enkephalin and monoamines, such as serotonin are known to be important mediators of acupuncture analgesia [14]. In this regard acupuncture analgesia is completely abolished in cats and mice by the injection of naloxone, a non-selective opioid antagonist [30, 31]. 
This suggests that acupuncture analgesia is mediated by endogenous opioid components of the descending pain modulating system. In addition, serotonin and norepinephrine are also known to be important neurochemical components involved in acupuncture analgesia [21, 33].

The formalin test is one of a number of widely used acute pain models [2, 15, 32]. Injection of diluted formalin into the plantar or dorsal surface of the paw produces a biphasic series of pain behaviors that included paw licking, biting, shaking, and flinching. Formalin-induced pain behavior is typically divided into two components (i.e. an early neuronal phase extending from the time of formalin injection to 10 min postinjection and a late inflammatory phase extending from $10 \mathrm{~min}$ to $1 \mathrm{hr}$ ). Moreover, the noxious input to the spinal cord resulting from formalin injection significantly increases spinal cord Fos expression [22]. Morphine [5] as well as electroacupuncture [6] reduces spinal Fos expression that was elevated by noxious stimulation. These results are consistent with a large body of literature indicating that spinal cord neuronal Fos protein expression can be used as an indicator of nociception.

The aim of this study was to determine whether BV administration has a suppressive effect on formalin-induced pain behavior and spinal Fos expression. In addition, we compared the antinociceptive effect of BV injection into the Zusanli acupoint with that obtained following injection into a non-acupoint.

\section{MATERIALS AND METHODS}

Animals: Experiments were performed on male Sprague Dawley rats weighing 200-250 g. All experimental animals were obtained from the Laboratory Animal Center of Seoul National University. They were housed in colony cages with free access to food and water. They were maintained in temperature and light controlled rooms $\left(23 \pm 0.5^{\circ} \mathrm{C}, 12 / 12\right.$ $\mathrm{hr} /$ light/dark cycle with lights on at 07:00) for at least 1 week prior to the study. All of the methods used in the present study were approved by the Animal Care and Use Committee at SNU and conform to NIH guidelines (NIH publication No. 86-23, revised 1985). The ethical guidelines of the International Association for the Study of Pain [36] for investigating experimental pain in conscious animals was also followed.

Drug treatment: BV of Apis mellifera (Sigma, St. Louis, MO, U.S.A.) at doses of 0.0016, 0.008, 0.016 and $0.08 \mathrm{mg} /$ $\mathrm{kg}$ was dissolved in physiological saline solution $(20 \mu \mathrm{l})$ and subcutaneously administered into the Zusanli acupoint located $5 \mathrm{~mm}$ lower and lateral to the anterior tubercle of tibia. A vehicle control group received an injection of a corresponding volume of saline solution into the same acupoint. The highest dose of BV $(0.08 \mathrm{mg} / \mathrm{kg})$ was used to compare the antinociceptive effects of injection into an acupoint with injection into a non-acupoint. To evaluate this, BV was injected into the Zusanli acupoint in one group of animals and into an arbitrary site located on the back (nonacupoint) in another group of rats $30 \mathrm{~min}$ prior to formalin injection.

Formalin test: Thirty minutes after BV injection, $1 \%$ formalin $(0.37 \%$ formaldehyde) in a volume of $20 \mu \mathrm{l}$ was injected subcutaneously into the plantar surface of the right hindpaw with a 30-G needle. Following formalin injection, the animals were placed in a temperature regulated plexiglas observation chamber. A mirror was attached underneath the chamber and was set at a $45^{\circ}$ angle to allow an unimpeded view of the animals' paws. Behavioral responses were then recorded using a video recording system. To access formalin-induced pain behavior, paw licking time was measured every $5 \mathrm{~min}$ during a $60 \mathrm{~min}$ post-injection period. Pawlicking time was divided into two components (i.e. an early neuronal phase extending from the time of formalin injection to $10 \mathrm{~min}$, phase 1 and a late inflammatory phase extending from $10 \mathrm{~min}$ to $60 \mathrm{~min}$, phase 2) and analyzed [15]. All behaviors were analyzed on the recorded videotapes by an observer who was blinded to the treatment group.

Fos immunohistochemistry: All procedures used in the present study are based on those described previously by Kwon et al. [24, 25]. Animals were perfused with calciumfree tyrode's solution followed by a fixative containing $4 \%$ paraformaldehyde and $0.2 \%$ picric acid in $0.1 \mathrm{M}$ phosphatebuffered saline (PBS, pH 6.9). Specimen was post-fixed by same fixative overnight and cryoprotected with $30 \%$ sucrose in PBS and sectioned on a cryostat (Microm, Germany). After elimination of endogenous peroxidase activity and preblocking with normal goat, the sections were incubated in polyclonal rabbit anti-Fos antiserum (Calbiochem, 1:10,000). The sections were processed with the avidinbiotin-peroxidase technique as previously described [24, 25]. Finally, Fos positive neurons were visualized using 3,3'-diaminobenzidine (DAB, Sigma Chemical Co., St. Louis, Mo, U.S.A.). Immunohistochemical controls consisted of sections incubated without primary or secondary antibodies.

Cell counting and image analysis: The selected sections were digitized with 4096 gray levels using a cooled CCD (Micromax Kodak 1317, Princeton instrument, U.S.A.) equipped with a computer-assisted image analysis system (Metamorph, Universal Imaging Co. U.S.A.). All data analysis procedures were performed blindly with respect to the experimental condition of the animal. To assess the effect of BV pretreatment on Fos expression in spinal neurons, four regions of the spinal cord were selected for examination based on cytoarchitectonic criteria. These four regions included the superficial lamina of the dorsal horn (SDH, lamina I-II), nucleus proprius (NP, lamina III-IV), the neck region of dorsal horn (NECK, lamina V-VI) and the ventral horn (VENT, lamina VII-IX).

Statistical analysis: One-way ANOVA was performed to determine the overall effect of BV pretreatment on formalin induced pain related behavior and on spinal Fos expression. Unpaired $t$-tests were used to determine the $\mathrm{p}$ value when ANOVA indicated a significant group difference. A p value $<0.05$ was considered statistically significant and all values 

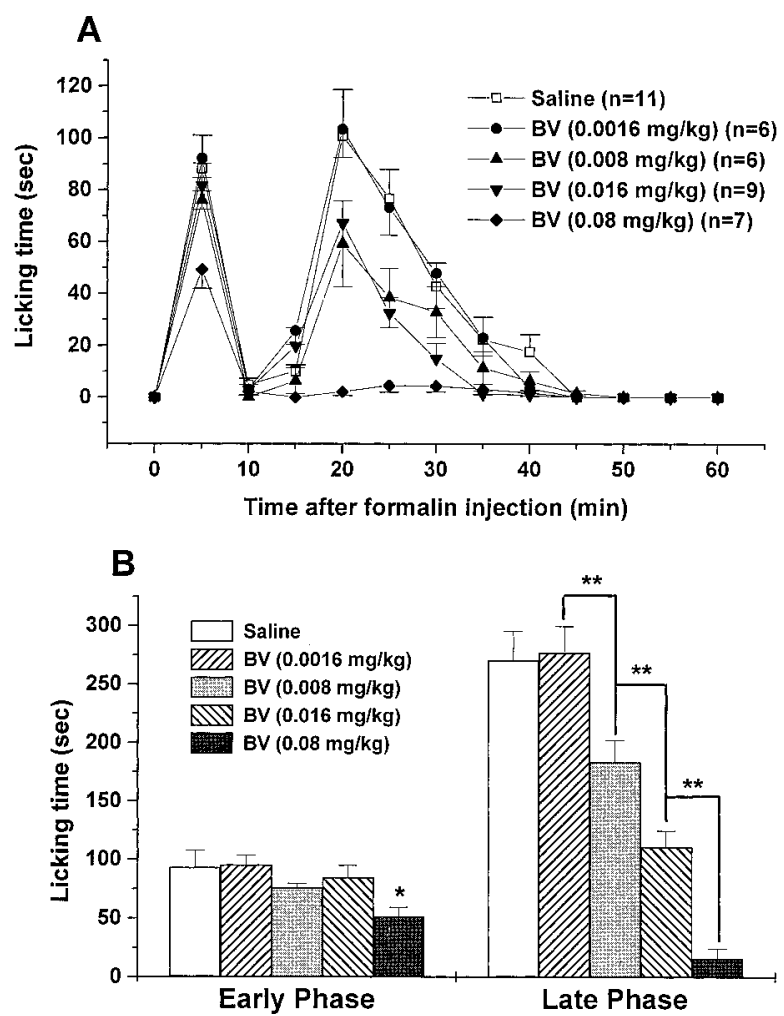

Fig. 1. A. Dose dependent effect of bee venom (BV) on formalininduced paw licking time. B. Formalin-induced paw licking time was divided into the two phases (i.e. the early and late phase) and analyzed. In the early phase, the highest dose (0.08 $\mathrm{mg} / \mathrm{kg}$ ) of BV suppressed the paw licking time induced by intraplantar formalin injection. The three lower doses of BV failed to inhibit the paw licking time in the early phase. In the late phase, $\mathrm{BV}$ dose dependently inhibited formalin-induced paw licking time. $* \mathrm{p}<0.05$ and $* * \mathrm{p}<0.01$, respectively.

are expressed as the mean \pm SEM.

\section{RESULTS}

Dose dependent effect of acupoint $B V$ pretreatment on formalin-induced pain behavior: In the early acute phase, BV injection into the Zusanli acupoint at doses of 0.0016, 0.008 , and 0.016 did not suppress formalin-induced pain behavior (Fig. 1A and B). Only the highest dose of BV significantly decreased the paw locking time in the early phase. In contrast during the late phase, BV injection in the Zusanli acupoint suppressed paw-licking time in a dose dependent manner. However, pretreatment with the lowest dose of BV $(0.0016 \mathrm{mg} / \mathrm{kg})$ failed to inhibit pain behaviors during the late phase.

Site comparison study: Based on the above results, the most effective dose of BV $(0.08 \mathrm{mg} / \mathrm{kg})$ was selected and then utilized for the acupoint versus nonacupoint comparison study. In the early phase, BV pretreatment into a nonacupoint on the back failed to block formalin-induced pain

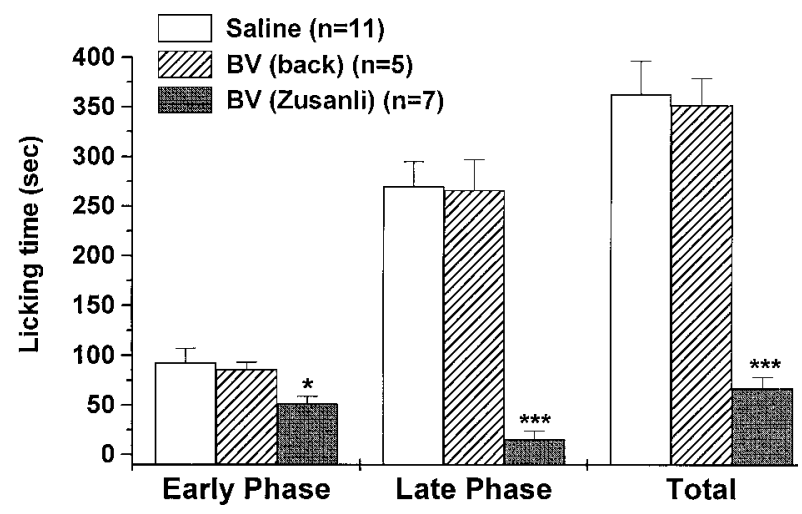

Fig. 2. This figure shows a comparison of bee venom (BV) injection into different sites on formalin-induced nociception. The dose dependent inhibitory effect of BV on the paw licking time induced by formalin was observed only in the late phase. BV injection into the Zusanli acupoint produced the greatest suppressive effect on the formalin-induced paw licking time. BV pretreatment into a non-acupoint on the back did not suppress the paw licking time evoked by formalin injection. BV injection into the Zusanli acupoint produced the greatest suppressive effect on the formalin-induced pain. $* \mathrm{p}<0.05$ and $* * * \mathrm{p}<0.001$, respectively.

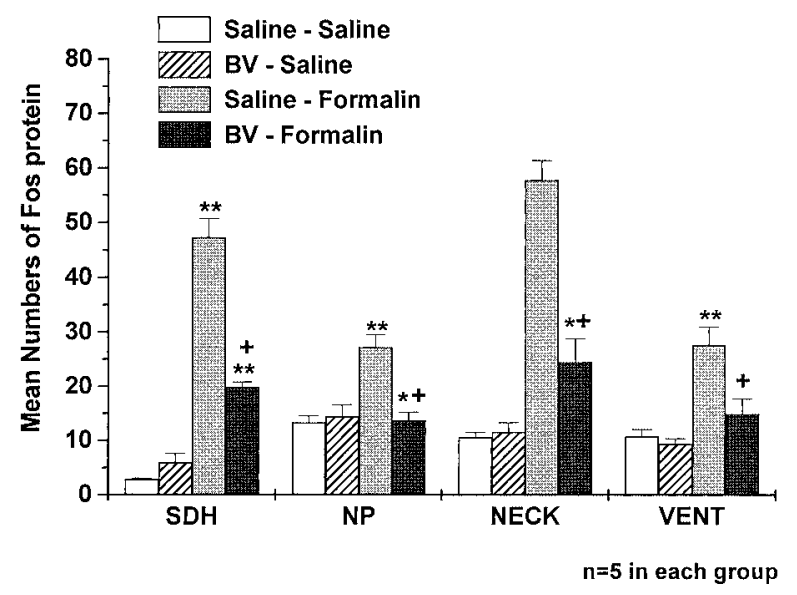

Fig. 3. This figure summarizes the effect of bee venom (BV) administration on formalin-induced spinal cord Fos expression at the lumbar L3-5 segments. There were no statistically significant differences between room control and the BV-saline group. BV treatment significantly suppressed the increased Fos expression induced by formalin injection. $* \mathrm{p}<0.05$ and $* * \mathrm{p}<0.01$ as compared with the saline-saline group, respectively. $+p<0.01$ as compared with saline-formalin group. (Abbreviations) SDH; superficial dorsal horn, NP; nucleus proprius, NECK; neck of dorsal horn, VENT; ventral horn.

behavior (Fig. 2). In contrast, BV injection into the Zusanli acupoint reduced paw licking time during phase 1 (* $\mathrm{p}<0.05)$. Moreover, BV pretreatment into the Zusanli acupoint also significantly suppressed formalin-induced paw licking time during the late phase $(* * * \mathrm{p}<0.001)$. BV pretreatment into an arbitrary site on the back (BV/back-Formalin) on the other hand showed no suppressive effect on 


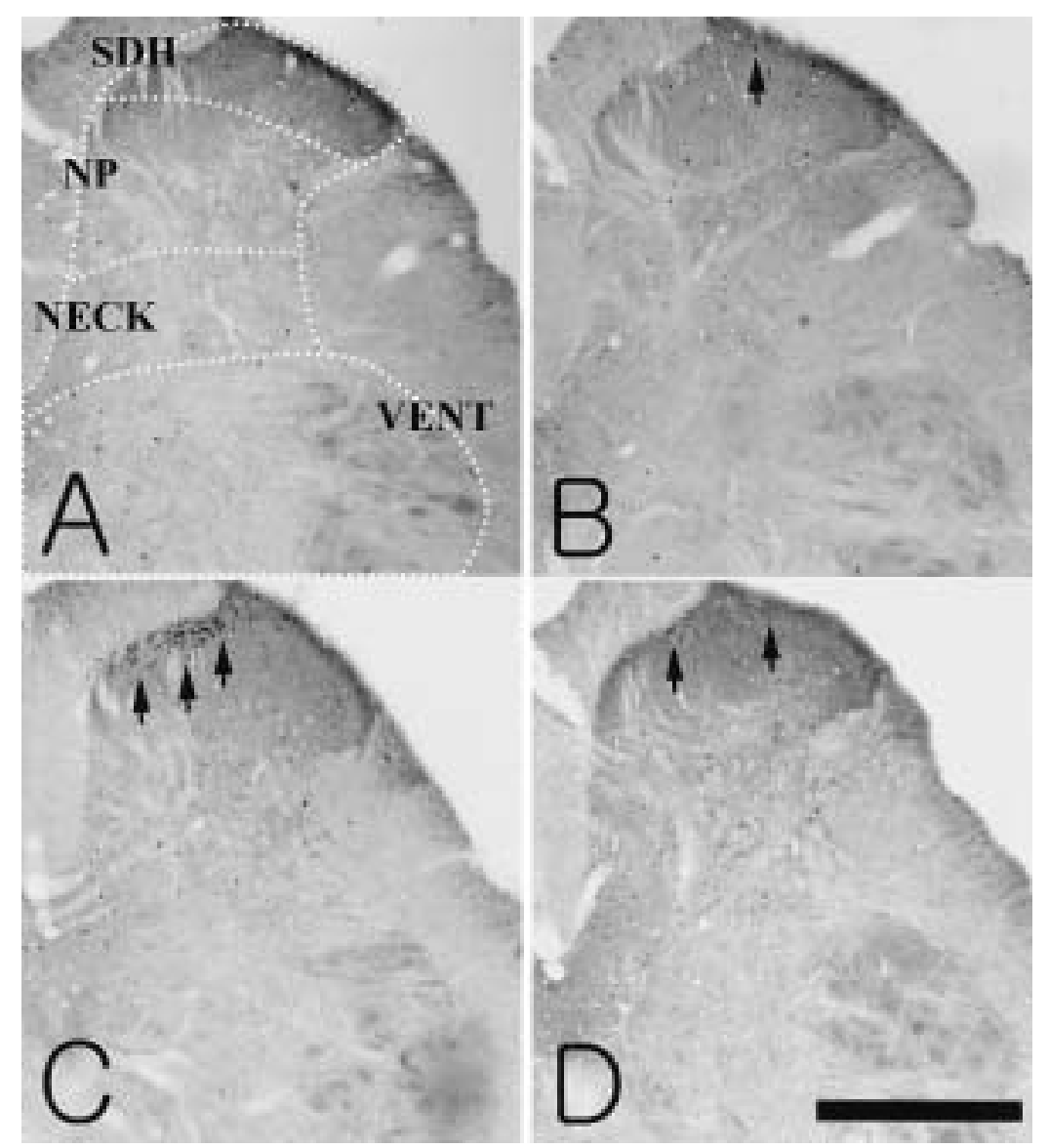

Fig. 4. Fos expression in the rat spinal cord from each treated animal. Although bee venom (BV) injection tended to increase Fos expression as compared with that of the saline-saline group, this increase was not significant (arrow in B). Intraplantar formalin injection dramatically and significantly increased Fos expression (arrows in C). The BV pretreated formalin group showed a significant decrease in spinal Fos expression as compared with that of the saline-formalin group (arrows in D). A saline-saline group. B BV-saline group. C saline-formalin group. D BV-formalin group. Scale bar $=500 \mu \mathrm{m}$. (Abbreviations) SDH; superficial dorsal horn, NP; nucleus proprius, NECK; neck of dorsal horn, VENT; ventral horn.

formalin-induced pain behavior as compared with the BVZusanli group.

Spinal Fos expression: Very few Fos positive neurons were observed in any region of the lumbar spinal cord (SDH, NP, NECK, and VENT in Figs. 3 and 4) in the salinesaline group. In the BV-saline treatment group, there was a slight but reproducible increase in the number of Fos positive neurons when compared with that of the saline-saline group, but this increase was not statistically significant. In the saline-formalin treatment group, Fos expression was significantly increased in every region of the lumbar spinal cord as compared with the saline-saline group $(* * \mathrm{p}<0.01)$. In contrast, BV pretreatment significantly decreased formalin-induced spinal Fos expression in the BV-formalin group as compared to saline pretreatment in the saline-formalin group $(* \mathrm{p}<0.05$ and $* * \mathrm{p}<0.01$, respectively).

\section{DISCUSSION}

Suppressive effect of $B V$ pretreatment on formalininduced pain behavior: It is well established that the early (acute) phase of formalin-induced pain behavior is produced by direct activation of primary afferent fibers, while pain behaviors associated with the late phase are associated with formalin-induced inflammatory reaction [31]. In the present study, we have shown that BV pretreatment at doses of $0.0016,0.008$, and $0.016 \mathrm{mg} / \mathrm{kg}$ did not reduce formalininduced paw licking time during the early phase (Fig. 1A and B). However the highest dose of BV $(0.08 \mathrm{mg} / \mathrm{kg})$ did suppress formalin-induced pain behavior during the early phase. In the late phase of the formalin test, BV pretreatment into the Zusanli acupoint produced a dose dependent reduction of formalin-induced paw licking time, except at 
the lowest dose tested $(0.0016 \mathrm{mg} / \mathrm{kg})$. Previously, Kwon and his co-workers demonstrated that $\mathrm{BV}$ administration produced both an antinociceptive and an anti-inflammatory effect on the acetic acid-induced writhing reflex in mice and on complete Freund's adjuvant-induced arthritis in rats [24, 25]. Based on these previous findings, we hypothesized that $\mathrm{BV}$ injected into an acupoint might behave as a chemical stimulant that activates the acupoint and subsequently engages the endogenous pain modulating system. Because whole BV contains stimulating components such as melittin and phospholipase $A_{2}$, it is possible that these stimulating components may activate the acupoint directly. Furthermore, we assumed that the antinociceptive effect of BV was from the melittin because it is major component of whole BV (50\% of dried BV) [26]. Thus we would propose that $\mathrm{BV}$ pretreatment into an acupoint produces its antinociceptive effect on formalin-induced pain by ultimately activating the endogenous pain modulation system. In the early phase, the highest dose of BV $(0.08 \mathrm{mg} / \mathrm{kg})$ suppressed the formalin-induced paw licking time, however lower doses of BV did not suppress this formalin-induced pain. This data would imply that the highest dose of BV was potent enough to rapidly activate the descending pain modulating system. However, lower doses of BV failed to produce the inhibitory effect on paw licking time in phase 1 .

In the late phase (phase 2), BV pretreatment into the Zusanli acupoint produced a dose dependent suppressive effect on formalin-induced pain behavior (except at the lowest dose of BV tested, $0.0016 \mathrm{mg} / \mathrm{kg}$ ). This low dose of BV might not be at a sufficient concentration to activate primary afferents and ultimately engage the descending pain modulating system. Acupuncture treatment has been reported to release enkephalin and dynorphin at the spinal cord level and these peptides are thought to directly inhibit afferent nociception [21]. Moreover acupuncture-evoked enkephalin release at midbrain levels is postulated to activate descending systems. These descending systems release serotonin and norepinephrine at the spinal cord level, which in turn block afferent pain transmission. Therefore it is possible that BV injection into an acupoint serves to activate descending systems that modulate nociceptive input at spinal cord levels via opioid, adrenergic and/or serotonergic mechanisms. In this regard a recent study of Kwon et al. [25] demonstrates that the antinociceptive effect of BV injected into Zhongwan acupoint on abdominal stretches is mediated by the $\alpha_{2}$ adrenergic system. However, further investigation is still necessary to elucidate the antinociceptive mechanisms induced by $\mathrm{BV}$ in the formalin test.

Acupoint vs non-acupoint stimulation: Kwon et al. reported that the BV injection into an acupoint produces a more potent antinociceptive effect than BV injection into a non-acupoint on the acetic acid-induced writhing reflex in mice [25]. It has also been reported that electroacupuncture (EA) stimulation of the Hoku acupoint suppresses tooth pulp stimulation-induced stress in rats [18]. In this latter study EA was administered to animals in the stressed state and the EA treated animals showed very little change in blood pressure compared to animals that were stressed but received EA in a non-acupoint on the tail. In addition, the EA treated animals had significantly lower blood levels of $\mathrm{ACTH}$ and corticosterone and the stress-induced cathecholamines norepinephrine, epinephrine and dopamine compared to the EA/non-acupoint treated group. In the present study we showed that BV injection into the Zusanli acupoint at the three highest doses tested produced a significant suppressive effect on pain behavior in the late phase of the formalin test. Conversely, BV pretreatment into a nonacupoint located on the back did not suppress the formalininduced pain behavior (Fig. 2). As alluded to above, we hypothesized that BV administration into an acupoint may activate the acupuncture-related neural circuit and through this activation, $\mathrm{BV}$ might produce its antinociceptive effect on formalin-induced pain behavior. We conclude from these results that the antinociceptive effect of BV is only produced when $\mathrm{BV}$ is administered into a specific acupoint.

The effect of $B V$ on spinal Fos expression and correlation relationship: It has been reported that peripheral noxious and non-noxious (tactile) stimulation increases Fos expression in a corresponding region of the spinal cord [22]. After the discovery that this immediate early gene could be used as a marker of nociceptive-related neurons, Fos protein has been used extensively to study spinal cord regions activated by nociception [19]. Gogas et al. [17] have previously demonstrated that formalin injection into plantar surface of hindpaw dramatically increases the spinal Fos expression and that intracerebroventricular injection of morphine dose dependently reduces spinal Fos expression in a naloxone reversible manner. Analgesic drugs such as dexamethasone [4] and aspirin [20] have also been shown to reduce spinal Fos expression induced by nociceptive stimuli.

In the present study, we divided the spinal cord into four regions (based in part on afferent fiber termination patterns) to allow regional quantitative analysis of Fos expression (Figs. 3 and 4). Among these four regions, the superficial dorsal horn has been shown to be an important area related to nociceptive processing in the formalin test [1]. Intraplantar injection of formalin dramatically increased spinal cord Fos expression. BV pretreatment into the Zusanli acupoint (BV-formalin) significantly suppressed the Fos expression that was evoked by formalin injection (Fig. 3). We initially hypothesized that BV injection alone would cause increased Fos expression in the spinal cord. Although Fos expression was slightly increased in the BV-saline group, there was no statistical difference as compared with that of the salinesaline group (Fig. 3). Thus if BV treatment is activating normal acupuncture related antinociceptive pathways in the central nervous system, the spinal involvement in this activation is not evident in Fos reacted spinal cords.

In this study, we provided evidence that BV pretreatment into the Zusanli acupoint suppresses formalin-induced pain behavior. In contrast BV treatment into a non-acupoint located on the back did not produce antinociceptive effects on formalin-induced pain behaviors. In addition, BV pretreatment into the Zusanli acupoint significantly suppressed 
spinal cord Fos expression evoked by formalin injection. These results suggest that BV injection into the Zusanli acupoint has an antinociceptive effect on formalin-induced pain.

ACKNOWLEDGMENT. This study was supported by a grant of the Korea Health 21 R\&D Project, Ministry of Health \& Welfare, Republic of Korea (01-PJ9-PG1-01CO01-0003). The publication of this manuscript was also supported by the Brain Korea 21 project in the College of Veterinary Medicine and School of Agricultural Biotechnology, Seoul National University.

\section{REFERENCES}

1. Abbadie, C., Taylor, B.K., Peterson, M.A. and Basbaum, A.I. 1997. Differential contribution of the two phases of the formalin test to the pattern of c-fos expression in the rat spinal cord: studies with remifentanil and lidocaine. Pain 69: 101-110.

2. Baamonde, A., Alvarez-Vega, M., Hidalgo, A. and Menendez, L. 2000. Effects of intraplantar morphine in the mouse formalin test. Jpn. J. Pharmacol. 83: 154-156.

3. Berman, B.M., Swyers, J.P. and Ezzo, J. 2000. The evidence for acupuncture as a treatment for rheumatologic conditions. Rheum. Dis. Clin. North. Am. 26: 103-115.

4. Buritova, J., Honore, P., Chapman, V. and Besson, J. 1996. Enhanced effects of co-administered dexamethasone and diclofenac on inflammatory pain processing and associated spinal c-Fos expression in the rat. Pain 64: 559-568.

5. Catheline, G., Le Guen, S. and Besson, J.M. 1999. Effects of opioid receptor antagonists on the effects of i.v. morphine on carrageenin evoked c-Fos expression in the superficial dorsal horn of the rat spinal cord. Brain Res. 824: 105-111.

6. Chang, C.J., Huang, S.T., Hsu, K., Lin, A., Stoller, M.L. and Lue, T.F. 1998. Electroacupuncture decreases c-fos expression in the spinal cord induced by noxious stimulation of the rat bladder. J. Urol. 160: 2274-2279.

7. Chen J., Luo, C. and Li, H.L. 1998. The contribution of spinal neuronal changes to development of prolonged, tonic nociceptive responses of the cat induced by subcutaneous bee venom injection. Eur. J. Pain 2: 359-376.

8. Chen, J., Luo, C., Li, H. and Chen, H. 1999. Primary hyperalgesia to mechanical and heat stimuli following subcutaneous bee venom injection into the plantar surface of hindpaw in the conscious rat: a comparative study with the formalin test. Pain 83: 67-76.

9. Chen, J., Li, H., Luo, C., Li, Z. and Zheng, J. 1999. Involvement of peripheral NMDA and non-NMDA receptors in development of persistent firing of spinal wide-dynamic-range neurons induced by subcutaneous bee venom injection in the cat. Brain Res. 844: 98-105.

10. Chen, H.S., Chen, J. and Sun, Y.Y. 2000. Contralateral heat hyperalgesia induced by unilaterally intraplantar bee venom injection is produced by central changes: a behavioral study in the conscious rat. Neurosci. Lett. 284: 45-48.

11. Chen, H.S. and Chen, J. 2000. Secondary heat, but not mechanical, hyperalgesia induced by subcutaneous injection of bee venom in the conscious rat: effect of systemic MK-801, a noncompetitive NMDA receptor antagonist. Eur. J. Pain 4: 389401.

12. Chen, H.S., Chen, J., Chen, J., Guo, W.G. and Zheng, M.H.
2001. Establishment of bee venom-induced contralateral heat hyperalgesia in the rat is dependent upon central temporal summation of afferent input from the site of injury. Neurosci. Lett. 298: 57-60.

13. Chen, J. and Chen, H.S. 2001. Pivotal role of capsaicin-sensitive primary afferents in development of both heat and mechanical hyperalgesia induced by intraplantar bee venom injeciton. Pain 91: 367-376.

14. Chung, S.H. and Dickenson, A. 1980. Pain, enkephalin and acupuncture. Nature (Lond.) 283: 243-244.

15. Dubuisson, D. and Dennis, S.G. 1977. The formalin test: a quantitative study of the analgesic effects of morphine, meperidine, and brain stem stimulation in rats and cats. Pain 4: 161174.

16. Ezzo, J., Hadhazy, V., Birch, S., Lao, L., Kaplan, G., Hochberg, M. and Berman, B. 2001. Acupuncture for osteoarthritis of the knee: a systematic review. Arthritis Rheum. 44: 819825 .

17. Gogas, K.R., Presley, R.W., Levine, J.D. and Basbaum, A.I. 1991. The antinociceptive action of supraspinal opioids results from an increase in descending inhibitory control: correlation of nociceptive behavior and c-fos expression. Neuroscience $\mathbf{4 2}$ : 617-628.

18. Han, S.H., Yoon, S.H., Cho, Y.W., Kim, C.J. and Min, B.I. 1999. Inhibitory effects of electroacupuncture on stress responses evoked by tooth-pulp stimulation in rats. Physiol. Behav. 66: 217-222.

19. Harris, J.A. 1998. Using c-fos as a neural marker of pain. Brain Res. Bull. 45: 1-8.

20. Honore, P., Buritova, J. and Besson, J.M. 1995. Aspirin and acetaminophen reduced both Fos expression in rat lumbar spinal cord and inflammatory signs produced by carrageenin inflammation. Pain 63: 367-375.

21. Hsu, D.T. 1996. Acupuncture. Reg. Anesth. 21: 361-370.

22. Hunt, S.P., Pini, A. and Evan, G. 1987. Induction of c-fos-like protein in spinal cord neurons following sensory stimulation. Nature (Lond.) 328: 632-634.

23. Koyama, N., Hirata, K., Hori, K., Dan, K. and Yokota, T. 2000. Computer-assisted infrared thermographic study of axon reflex induced by intradermal melittin. Pain 83: 133-139.

24. Kwon, Y.B., Lee, J.D., Lee, H.J., Han, J.H., Mar, C.W., Kang, S.K., Beitz, A.J. and Lee, J.H. 2001. Bee venom injection into an acupuncture point reduces arthritis associated edema and nociceptive responses. Pain 90: 271-280.

25. Kwon, Y.B., Kang, M.S., Han, H.J., Beitz, A.J. and Lee, J.H. 2001. Visceral antinociception produced by bee venom stimulation of the Zhongwan acupuncture point in mice: role of alpha(2) adrenoceptors. Neurosci. Lett. 308: 133-137.

26. Lariviere, W.R. and Melzack, R. 1996. The bee venom test: a new tonic-pain test. Pain 66: 271-277.

27. Li, K.C., Zheng, J.H., and Chen, J. 2000. Involvement of spinal protein kinase $\mathrm{C}$ in induction and maintenance of both persistent spontaneous flinching reflex and contralateral heat hyperalgesia induced by subcutaneous bee venom in the conscious rat. Neurosci. Lett. 285: 103-106.

28. Luo, C., Chen, J., Li, H.L. and Li, J.S. 1998. Spatial and temporal expression of c-Fos protein in the spinal cord of anesthetized rat induced by subcutaneous bee venom injection. Brain Res. 806: 175-185.

29. Pomeranz, B. and Cheng, R. 1979. Suppression of noxious responses in single neurons of cat spinal cord by electroacupuncture and its reversal by the opiate antagonist naloxone. Exp. Neurol. 64: 327-341. 
30. Pomeranz, B. and Paley, D. 1979. Electroacupuncture hypalgesia is mediated by afferent nerve impulses: an electrophysiological study in mice. Exp. Neurol. 66: 398-402.

31. Tjolsen, A., Berge, O.G., Hunskaar, S., Rosland, J.H. and Hole, K. 1992. The formalin test: an evaluation of the method. Pain 51: 5-17.

32. Ulett, G.A., Han, J. and Han, S. 1998. Traditional and evidence-based acupuncture. South. Med. J. 91: 1115-1120.

33. Zheng, J.H., and Chen, J. 2000. Modulatory roles of the ade- nosine triphosphate $\mathrm{P} 2 \mathrm{x}$-purinoceptor in generation of the persistent nociception induced by subcutaneous bee venom injection in the conscious rat. Neurosci. Lett. 278: 41-44.

34. Zheng, J.H. and Chen, J. 2001. Differential roles of spinal neurokinin $1 / 2$ receptors in development of persistent spontaneous nociception and hyperalgesia induced by subcutaneous bee venom inejction in the conscious rat. Neuropeptides 35: 32-44.

35. Zimmermann, M. 1983. Ethical guidelines for investigations of experimental pain in conscious animals. Pain 16: 109-110. 